\title{
Local and global visual mechanisms underlying individual differences in the rod-and-frame illusion
}

\author{
DONATELLA SPINELLI and GABRIELLA ANTONUCCI \\ University of Rome "La Sapienza," Italy and Clinica S. Lucia, Rome, Italy \\ ROBERTA DAINI \\ University of Rome "La Sapienza," Rome, Italy \\ and \\ PIERLUIGI ZOCCOLOTTI \\ University of Rome "La Sapienza," Italy and Clinica S. Lucia, Rome, Italy
}

\begin{abstract}
The role of local and global visual mechanisms in individual differences in the rod-and-frame (RF) effect was investigated. Field-dependent and field-independent observers, selected on the basis of Witkin and Asch's (1948) classical procedure, were submitted to the small RF test (Coren \& Hoy, 1986). Four frame tilts and two gap sizes were used. As expected, direct effects (i.e., rod settings in the direction of frame tilt) were observed at small degrees of frame tilt, while indirect effects (i.e., rod settings in the direction opposite that of frame tilt) were observed at larger frame tilts. Fielddependent observers showed larger direct effects in the case of the small gap. Indirect effects were comparable in both field-dependent and field-independent subjects, regardless of gap size. Following the model proposed by Wenderoth and Johnstone (1987), these findings indicate that low-level visual mechanisms, responsible for local orientation interactions, have a different gain in fielddependent and field-independent individuals. In contrast, global visual mechanisms, presumably acting by means of long-range mechanisms, do not distinguish between these two subgroups.
\end{abstract}

Although the apparent vertical is indicated with remarkable precision by an upright observer, errors become greater when a tilted frame is added in the dark (the rod-and-frame [RF] effect). Individual differences in the RF effect may be extremely large, characterizing observers as either field dependent or field independent (Asch \& Witkin, 1948; Witkin \& Asch, 1948). Starting from a cognitive-style conception of these differences, a large number of studies have focused on the individual correlates of the field dependence-field independence dimension (for a review, see Witkin \& Goodenough, 1981).

In recent years, research on RF effects has concentrated less on the problem of individual differences and has focused instead on the mechanisms responsible for the effect. Several studies have shown that visual-vestibular interactions are at work in the RF effect (e.g., Goodenough, Cox, Sigman, \& Strawderman, 1985). In the presence of a tilted RF display, an objectively upright observer feels and behaves as if he or she were tilted in the direction opposite that of frame tilt (e.g., Goodenough, Sigman, Olt-

This research was supported by Consiglio Nazionale delle Ricerche and Ministero della Pubblica Istruzione grants to D.S. and P.Z. Address correspondence to D. Spinelli, Department of Psychology, via dei Marsi 78,00185, Rome, Italy (e-mail: dippsi39@irmunisa.bitnet). man, Rosso, \& Mertz, 1979). Consequently, at least part of the.illusion is due to a compensatory shift of the subjective vertical in the direction opposite that of experienced body tilt.

However, several studies have shown the presence of distortion of rod orientation without the intervention of visuo-vestibular mechanisms (e.g., with frame tilts of $0^{\circ}$; Goodenough, Oltman, Sigman, Rosso, \& Mertz, 1979). In these cases, purely visual mechanisms, such as those at work in the traditional visual orientation illusion (e.g., the Zöllner illusion), are at work. These visual orientationcontrast mechanisms have been most frequently studied with small frames $\left(<10^{\circ}\right)$, in comparison with the $28^{\circ}-40^{\circ}$ frame size typically used in previous studies examining individual differences. Variations in frame size cause two important differences in the illusion: (1) A large frame produces much larger rod-setting errors than a small frame (e.g., Ebenholtz \& Callan, 1980), as well as only so-called direct effects (i.e., tilt of the apparent vertical in the direction of frame tilt, or positive values); and (2) a small frame produces both direct and indirect effects (i.e., tilt of the apparent vertical in the direction opposite that of frame tilt, or negative values), depending on the degree of frame tilt (e.g., Wenderoth \& Beh, 1977). The transition from direct to indirect effects is due to the ambiguous orientation of the squared frame. With increasing tilt, this appears to shift from a square tilted in one 
direction to a diamond tilted in the opposite direction. According to Beh, Wenderoth, and Purcell (1971), the axis of symmetry of the figure closest to the vertical determines the perceived orientation of the figure and influences the perception of the vertical. At small tilts, the dominant axis is the one parallel to the two tilted sides of the square; at larger tilts (i.e., between $22^{\circ}$ and $45^{\circ}$ ), the diagonal of the inducing figure is closer to the verti$\mathrm{cal}$, and therefore becomes the dominant axis. Since it is tilted on the opposite side of the previous reference, it produces errors in the direction opposite to that of frame tilt. In this view, the effect of the frame may be considered as the result of the interaction between the apparent orientation of the inducing figure axes with that of the rod. This led Wenderoth and coworkers to consider the $\mathrm{RF}$ effect as a two-dimensional (2-D) orientation illusion, an analog to the 1-D tilt illusion and tilt aftereffect. Results obtained with 2-D crossed gratings (plaids; Johnstone \& Wenderoth, 1989a; Wenderoth \& Van der Zwan, 1991) were consistent with this idea; thus, the angular function obtained with this stimulus was similar to that of small RF displays, showing both direct and indirect effects.

On the basis of their own findings, as well as on neurophysiological data on the cortical visual areas (e.g., Allman, Miezin, \& McGuinness, 1985), Wenderoth and Johnstone $(1987,1988 \mathrm{~b})$ proposed that direct effects arise from features of the stimulus that are processed both by low and high levels of the visual system, while indirect effects involve only higher mechanisms. At the low level, local interaction between orientations takes place (presumably at the level of the V1 neuron classical receptive field). At higher levels of visual processing, remote interactions between contours take place, and global features of the figure (such as symmetry) can be analyzed. This might happen in extrastriate areas, where neurons have a larger receptive field and can also be activated by illusory contours (or perhaps also in striate areas-long-range interactions have also been reported in V1, involving the total receptive field of the neuron; T'so, Gilbert, \& Wiesel, 1986). This global processing may encode a right-tilted square frame as a lefttilted diamond (Wenderoth \& Johnstone, 1987).

Several psychophysical findings are consistent with this model. ${ }^{1}$ Thus, the direct effect is reduced by manipulation designed to reduce local mechanism contributionfor instance, by increasing the distance between the test and inducing figure (gap), both with plaids (Johnstone \& Wenderoth, 1989) and with an RF display (Coren \& Hoy, 1986; Zoccolotti, Antonucci, \& Spinelli, 1993). In contrast, the indirect effects either are not influenced by the manipulation of this parameter (Wenderoth \& Johnstone, 1988b) or they are influenced in the opposite direction (Wenderoth, van der Zwan, \& Johnstone, 1989; Zoccolotti et al., 1993).

The model proposed by Wenderoth and coworkers is based on studies using only small 1-D or 2-D displays. However, the same mechanisms may also be suitable for addressing the perceptual illusion with a large RF dis- play (Johnstone \& Wenderoth, 1989). Since field dependence is an important individual property measured with a large RF display, it would be interesting to investigate whether this dimension is also measured with a small RF display, and, if so, to assess which mechanisms underlie individual differences according to the model. Thus, one objective of the present research was to investigate whether the field-dependence dimension is involved in the case of a small visual inducing display. The subjects were selected according to their degree of field dependence, measured using the traditional Witkin and Asch (1948) procedure. Only those observers who were either very field dependent or very field independent were selected. They were then presented with a small RF display.

To date, individual differences with small and large RF displays have been investigated only as part of a large factor-analytic study (Goodenough, Oltman, \& Cox, 1987). Of relevance to the present study is the finding that the performance on the standard RF test was only moderately related to rod-adjustment errors induced by small frames. It was proposed that both visual-vestibular and orientation-contrast mechanisms contribute to performance on the standard RF test; on the other hand, performance on small RF displays would only depend on orientation-contrast mechanisms. However, since a single frame tilt was used $\left(15^{\circ}\right)$, this study does not allow for the separate examination of direct and indirect effects, which appear to be the distinguishing characteristics with small inducing displays.

According to Wenderoth and Johnstone's (1987) model, the relative size of direct and indirect effects in field-dependent and field-independent subjects might provide information about the level of the neural mechanism responsible for the field-dependence dimension. If field dependence is at least partially determined at low-level visual processing, we should expect larger direct (but not indirect) effects in field-dependent individuals. In contrast, if it depends on higher level processing, we should expect a major increment in the percentage of indirect effects, which are entirely determined at this level. If both mechanisms are involved, we should expect an increment of both effects (larger for the direct, which depends on both mechanisms). In contrast, if visuo-vestibular mechanisms are the only determinants of the illusion in the standard apparatus, no difference should be expected in the experimental condition, which should minimize or abolish these mechanisms. Finally, since the size of direct and indirect effects is differently modulated by the gap, we investigated whether the gap size has similar or different effects in field-dependent and field-independent individuals.

\section{METHOD}

\section{Subjects}

Performance on the classical RF display was used to select field-dependent and field-independent subjects. Sixty-four 20 30 -year-old volunteers participated in the study. All subjects had normal or corrected-to-normal vision. 
The RF device used had 96-cm-long sides. A 91.5-cm-long rod was used. The frames and the rod were made of a 1.2-cm-wide luminescent tape and were the only visible elements in a darkened room. The frames and the rod were coplanar. The observer was located at a distance of $141 \mathrm{~cm}$ from the stimulus. The subject's head was supported by a headrest.

Two frame tilts were used $\left(28^{\circ}\right.$ counterclockwise [CCW] and $28^{\circ}$ clockwise $\left.[\mathrm{CW}]\right)$, with two rod starting positions $\left(28^{\circ} \mathrm{CCW}\right.$ and $28^{\circ} \mathrm{CW}$ ). This produced a total of four visual displays. Each display was presented twice, for a total of eight trials. The subjects were instructed to direct the experimenter in aligning the rod with the gravitational vertical. The method of adjustment was used. Bracketing was permitted, and no time limit was given.

To select field-dependent and field-independent subjects, the algebraic mean of the rod-setting errors from the vertical was calculated. Subjects were considered field independent if their mean error was below $1.5^{\circ}$ of error, and field dependent if their mean was above $4^{\circ}$ of error, according to previously established criteria (Pizzamiglio \& Zoccolotti, 1981). Out of the entire group of 64 subjects, 43 (20 field dependent and 23 field independent) were within these limits. Because of various problems, the final sample included 15 field-dependent and 15 field-independent observers. The mean rod-setting errors for the two selected groups were 6.43 $(S D=2.1)$ for field-dependent subjects and $.67(S D=.6)$ for field-independent subjects.

\section{Stimuli}

As in Coren and Hoy's (1986) procedure, the stimuli were printed by a laserwriter. The viewing distance was $57 \mathrm{~cm}$, at which $1 \mathrm{~cm}$ subtends $1^{\circ}$ at the observer's eye. Each side of the square was $6 \mathrm{~cm} \times 1 \mathrm{~mm}$. The use of this paradigm allows the length of the rod to be easily changed, while maintaining a constant gap across conditions. ${ }^{2}$ The rods ( $1 \mathrm{~mm}$ wide) were of two different lengths $-5.2 \mathrm{~cm}$ and $1.8 \mathrm{~cm}$ when the displays were in the vertical position $\left(0^{\circ}\right)$, producing two gaps of 0.3 and $2 \mathrm{~cm}\left(20^{\prime}\right.$ and $2^{\circ}$ ), respectively. In all cases, the rods were $1 \mathrm{~mm}$ wide. In order to maintain a constant gap, the rod varied in length with the different tilts of the display: For the small gap condition, the rod varied between $5.1 \mathrm{~cm}$ and $6.5 \mathrm{~cm}$; for the large gap condition, it varied between $1.75 \mathrm{~cm}$ and $3.1 \mathrm{~cm}$. The stimuli were presented inside a 16-cm-wide circular window in the frontal plane.

The frame was presented at $0^{\circ}, 11^{\circ}, 22^{\circ}$, and $33^{\circ}$, both $\mathrm{CW}$ and $\mathrm{CCW}$, for a total of 7 different tilts. The rods were presented in 13 different tilts from $6^{\circ} \mathrm{CCW}$ to $6^{\circ} \mathrm{CW}$ in $1^{\circ}$ steps for each display condition. This produced 91 stimuli for each gap condition, for a total of 182 stimuli.

\section{Procedure}

Each subject took part in a single 1-h session, with a short rest period after half of the stimuli had been presented. The subject was seated on a chair with his or her head on a headrest. The experiment was carried out in a uniformly lit room. The stimuli were presented in the same random sequence for all subjects. A forcedchoice procedure was used. The subjects were instructed to judge whether the rod appeared to be tilted in a $\mathrm{CW}$ or a $\mathrm{CCW}$ direction. No time limit was given.

\section{RESULTS}

As in Coren and Hoy's (1986) study, results of the small RF were analyzed using the unidimensional Guttman Scale. The subjects' responses were analyzed separately for the two gap conditions (small and large) and for each display orientation $\left(0^{\circ}, 11^{\circ}, 22^{\circ}\right.$, and $33^{\circ}$ $\mathrm{CW}$, and $11^{\circ}, 22^{\circ}$, and $33^{\circ} \mathrm{CCW}$ ), for a total of 14 different conditions. The coefficients of reproducibility have a median value of .966 (range $=.907-1)$ for the field-independent subgroup and a median value of .914 (range $=.835-.969)$ for the field-dependent subgroup.

Preliminary analyses showed no difference between $\mathrm{CW}$ and $\mathrm{CCW}$ orientations. For the purpose of analysis, therefore, rod settings were marked positive when they were in the same direction as frame tilt, and negative when they were in the opposite direction, for both the $\mathrm{CW}$ and the $\mathrm{CCW}$ display orientations.

The mean rod settings as a function of frame tilt, group, and gap size are presented in Figure 1. The results confirm a number of previous findings. First, as expected, rod-setting errors are considerably smaller in this condition than are those observed with the large RF display (e.g., Coren \& Hoy, 1986). Second, the direction of the effect varies with degree of frame tilt: The effects are direct (i.e., in the direction of frame tilt) for small frame tilts $\left(11^{\circ}\right)$, and become indirect (i.e., away from frame tilt) at the largest frame tilt $\left(33^{\circ}\right)$ (Wenderoth \& Beh, 1977). Third, in the case of a small gap, the curve is generally more in the direction of frame tilt than it is with a larger gap; consequently, in the latter case, direct
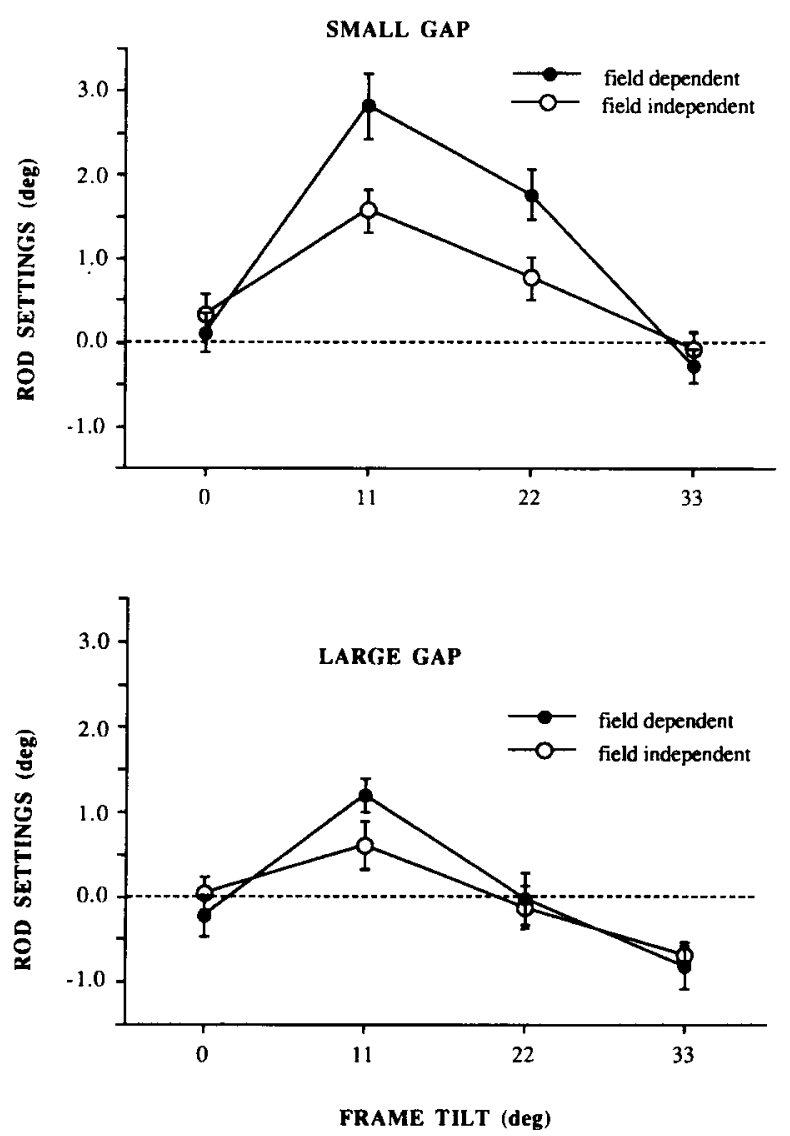

Figure 1. Rod-setting errors as a function of the frame tilts for small ( $20^{\prime}$ of arc) and large ( $2^{\circ}$ of arc) distances between test and inducing figure. Mean data of field-dependent and field-independent subjects are represented by filled and open circles, respectively. Positive values represent direct effects; negative values represent indirect effects. Vertical bars represent standard errors about the mean. 
effects are smaller and indirect effects are more pronounced than they are with the small gap (Zoccolotti et al., 1993).

The specific purpose of the study was to examine performance on a small RF device as a function of individual differences along the field-dependence dimension. For this purpose, the mean rod settings were submitted to an analysis of variance, with group (field dependent or field independent) as an unrepeated measure and gap (small or large) and frame orientation $\left(11^{\circ}, 22^{\circ}\right.$, or $\left.33^{\circ}\right)$ as repeated factors. Data relative to the $0^{\circ}$ tilt condition were not included in the analysis because they might have artificially inflated interactions with the orientation factor.

The analysis indicated a main effect of gap $[F(1,28)=$ $74.2, p<.0001]$ and of frame orientation $[F(2,56)=$ $67.2, p<.0001]$. The main effect of the group factor was not significant $[F(1,28)=2.46$, n.s. $]$. The group $\times$ gap interaction was significant $[F(1,28)=4.06, p=.05]$; analysis of simple effects indicated that the dependent group had greater effects than did the independent group in the small-gap condition $[F(1,28)=4.25, p<.05]$ but did not in the large-gap condition $(F<1)$. The group $\times$ frame-orientation interaction was significant $[F(2,56)=$ $5.14, p<.01]$; the difference between the two groups was significant at $11^{\circ}$ of frame orientation $[F(1,28)=$ $5.9, p<.05]$, but not at $22^{\circ}$ or $33^{\circ}$. The gap $\times$ frameorientation interaction was significant $[F(2,56)=9.21$, $p<.01$ ]; although always significant (in all cases $p<$ .0001 ), the difference between the small- and large-gap conditions was smaller at $33^{\circ}$ of frame orientation $\left(0.58^{\circ}\right)$ than at $11^{\circ}$ or $22^{\circ}\left(1.29^{\circ}\right.$ and $1.35^{\circ}$, respectively $)$.

The second-order group $\times$ gap $\times$ frame-orientation interaction was also significant $[F(2,56)=3.23, p<.05$; see Figure 1 [top and bottom]). In the top part of Figure 1, data of field-dependent and field-independent subjects are presented as a function of frame tilt for small gap size. The general shape of the two curves is similar. However, larger direct effects were observed for field-dependent subjects at $11^{\circ}(t=2.592, p<.025)$ and $22^{\circ}(t=2.437, p<.05)$ of frame tilt. The two groups did not differ at the $33^{\circ}$ tilt. In the bottom part of Figure 1, results are presented for the large-gap condition. The curves for the two groups have a similar trend, with a clear indirect effect at $33^{\circ}$. The difference between subjects was not significant for any of the three conditions.

In order to evaluate the sensitivity of the individual classification on the basis of the small RF test, we examined the two conditions in which a significant difference was observed between the two groups (i.e., $11^{\circ}$ and $22^{\circ}$, with small gap). In both cases, several field-dependent observers showed illusory errors well within the range of the field-independent observers. For example, in the case of the $11^{\circ}$ frame-tilt, small-gap condition, 5 subjects out of 15 made a mean rod-setting error that was smaller than the mean value of field-independent individuals. It therefore appears that the use of the small RF display to determine individual value along the field dependence-field independence dimension leads to a relatively large number of misclassifications with respect to the traditional RF display.

\section{DISCUSSION}

In general, the present results are consistent with those of Goodenough et al. (1987). In spite of the similarity between stimulus elements, data on the large and small RF displays show only a moderate overlap. In agreement with classical findings, the RF effect-in the dark, with large, tilted frame - induces larger individual differences, with some subjects showing large rod-setting errors and others showing almost no illusory effect. In comparison, the effects observed using the small RF display - presented with the lights on-were small, and reduced the error ratio between field-dependent and fieldindependent subjects from a factor of 10 to a factor of no more than two. According to Goodenough et al. (1987), this difference between the two similar visual displays is due to visuo-vestibular mechanisms. These are active only in the case of the large display, and inflate the rodsetting errors seen among field-dependent subjects. It therefore appears that Wenderoth and Johnstone's (1987) model should also include visual-vestibular mechanisms, in order to provide a general framework to account for the RF effect.

In the case of the small RF display, it seems relevant that the two groups of subjects did not show differences in all frame conditions. Individuals classified as field dependent showed greater illusory direct effects with the small RF display, limited to the case of a small gap; in contrast, indirect effects were comparable to those observed in field-independent subjects for both gap sizes. These findings indicate that the mechanisms responsible for direct (but not indirect) effects have different gain in field-dependent and field-independent observers.

According to Wenderoth and Johnstone's (1987) model, direct effects reflect the activity of both low- and high-level visual processing, and indirect effects reflect the activity of only high-level visual processing. At this level, global analysis of figures takes place, the illusory contours are analyzed, and long-range interactions occur. No difference between field-dependent and fieldindependent subjects seems to occur at this global level. In fact, indirect effects were the same in the two groups, indicating that the difference between field-dependent and field-independent individuals occurs only at lowlevel visual processing. Consistent with this view is the finding that differences between field-dependent and field-independent subjects were shown only in the smallgap condition favoring local interactions. When the test figure and the inducing figure are relatively far apart, the interactions between their orientations diminish, as suggested by the literature on tilt illusion (e.g., Carpenter \& Blakemore, 1973). The persistence of direct effects of about the same size as the indirect effects in the largegap condition-when local processes are removedmay indicate the residual effect of a global processing of 
the figure (Wenderoth \& Johnstone, 1987). Lack of difference in this condition between field-dependent and field-independent groups for direct effects is consistent with the idea that high-level global processing does not distinguish between these two groups of subjects.

In summary, considering the results of this study and those of previous studies reported in the literature, three separate mechanisms seem to be involved in individual performance on the standard version of the RF effect. (1) The role of visual-vestibular interactions (not studied in the present paper) has been clearly documented in the case of large frames presented in the dark (e.g., Goodenough et al., 1985). These mechanisms have different gain in field-dependent and field-independent subjects (i.e., the errors are larger among field-dependent subjects). (2) Local orientation interactions contribute to the illusory effect as well. Contrary to early hypotheses, they are present not only with small frames, but also occur in the case of large frames (Goodenough, Oltman, et al., 1979); what seems critical for activating these mechanisms is the distance between the inducing and the test figure. It may be noted that gap size is not commonly reported in studies on the field dependence-field independence dimension, and that it may vary greatly in different laboratories. These mechanisms have different gain in field-dependent and field-independent observers; errors are larger in field-dependent observers. The presence of a difference in local visual processing between field-dependent and field-independent individuals is consistent with the previous observation (Immergluck, 1966) that field-dependent individuals are more susceptible to Zöllner's geometric illusion, where short-range interactions are important. (3) The third mechanism involved is global figure processing. In the case of small frames (particularly with a large gap), indirect effects are obtained with large frame tilts, presumably because of the observers' reliance on the virtual axis of symmetry defined by the diagonal of the frame. In the case of large frames, indirect effects are not commonly observed because they are masked by larger visual-vestibular effects; however, the angular function of the illusion indicates that the illusion decreases after $20^{\circ}$ of frame tilt (Zoccolotti, Antonucci, Goodenough, Pizzamiglio, \& Spinelli, 1992), a finding that is incongruous with the visuo-vestibular hypothesis, but that is easily explained by global analysis of the figure. Thus, it seems reasonable that, for frame tilts of between $22^{\circ}$ and $45^{\circ}$ (such as in the classical procedure, where a value of $28^{\circ}$ is commonly used), global factors act by reducing the effect produced by visuo-vestibular interactions, and they act in the same direction for smaller frame tilts. With regard to the field dependence-field independence dimension, it is interesting that the global high-level mechanisms do not have a different gain in field-dependent subjects.

In conclusion, this study, together with that of Goodenough et al. (1987), indicates that individual differences in field dependence/independence may be determined at early levels of visual processing (i.e., through visual-vestibular interaction and visual local mecha- nisms). The link between these mechanisms and the personality traits that have been associated with this dimension is still not understood. One possible clue may be provided by the evolutionary view of field dependence proposed by Witkin and colleagues, which states that whenever greater ability in orientation perception has a critical value in adjusting to a specific environment, this may be associated with other psychological traits specifically selected within the same population. Crosscultural and behavioral genetic research provides some support for this statement (for reviews, see Witkin, 1978; Witkin \& Goodenough, 1981).

\section{REFERENCES}

Allman, J. M., Miezin, F., \& McGuinness, E. (1985). Stimulus specific responses from beyond the classical receptive field: Neurophysiological mechanisms for local-global comparisons in visual neurones. Annual Review of Neuroscience, 8, 407-430.

Asch, S. E., \& WiTkIN, H. A. (1948). Studies in space orientation: I. Perception of the upright with displaced visual fields. Journal of Experimental Psychology, 38, 325-337.

Beh, H. C., Wenderoth, P. M., \& Purcell, A. T. (1971). The angular function of a rod-and-frame illusion. Perception \& Psychophysics, 9(4), 353-355.

Carpenter, R. H. S., \& Blakemore, C. (1973). Interactions between orientations in human vision. Experimental Brain Research, 18, 287 303.

CoRen, S., \& Hoy, V. S. (1986). An orientation illusion analog to the rod and frame: Relational effects in the magnitude of the distortion. Perception \& Psychophysics, 39, 159-163.

EBENHOLTZ, S. M. (1977). Determinants of the rod and frame effect: The role of retinal size. Perception \& Psychophysics, 22, 531-538.

Ebenholtz, S. M., \& Callan, J. W. (1980). Modulation of the rod and frame effects: Retinal angle vs. apparent size. Psychological Research, 42, 327-334.

Goodenough, D. R., Cox, P. W., Sigman, E., \& Strawderman, W. E. (1985). A cognitive-style conception of the field-dependence dimension. Cahiers de Psychologie Cognitive, 5, 687-706.

Goodenough, D. R., Oltman, P. K., \& Cox, P. W. (1987). The nature of individual differences in field dependence. Journal of Research in Personality, 21, 81-99.

Goodenough, D. R., Oltman, P. K., Sigman, E., Rosso, J., \& Mertz, H. (1979). Orientation contrast effects in the rod-and-frame test. Perception \& Psychophysics, 25, 419-424.

Goodenough, D. R., Sigman, E., Oltman, P. K., Rosso, J., \& Mertz, H. (1979). Eye torsion in response to a tilted visual stimulus. Vision Research, 19, 1177-1179.

IMMERGLUCK, L. (1966). Resistance to an optical illusion, figural after-effects, and field dependence. Psychonomic Science, 6, 281-282.

Johnstone, S., \& Wenderoth, P. (1989). Spatial and orientation specific integration in the tilt illusion. Perception, 18, 5-23.

Pizzamiglio, L., \& ZoccolotTi, P. (1981). Sex and cognitive influence on visual hemifield superiority for face and letter recognition. Cortex, 17, 215-226.

T'so, D. Y., Gilbert, C. D., \& WiEsel, T. N. (1986). Relationships between horizontal interactions and functional architecture in cat striate cortex revealed by cross-correlational analysis. Journal of Neuroscience, 6, 1160-1170.

WENDEROTH, P., \& BEH, H. (1977). Component analysis of orientation illusions. Perception, 6, 57-75.

Wenderoth, P., \& Johnstone, S. (1987). Possible neural substrates for orientation analysis and perception. Perception, 16, 693-709.

WENDEROTH, P., \& JOHNSTONE, S. (1988a). The differential effects of brief exposures and surrounding contours on direct and indirect tilt illusion. Perception, 17, 165-176.

WENDEROTH, P., \& Johnstone, S. (1988b). The different mechanisms of the direct and indirect tilt illusions. Vision Research, 28, 301-312. WENDEROTH, P., \& VAN DER ZWAN, R. (1991). Local and global mech- 
anisms of one- and two-dimensional orientation illusions. Perception \& Psychophysics, 50, 321-332.

Wenderoth, P., VAN DER ZWAN, R., \& Johnstone, S. (1989). Orientation illusion by briefly flashed plaids. Perception, 18, 715-728.

WIT KIN, H. A. (1978), Cognitive styles in personal and cultural adaptation. Worcester, MA: Clark University Press.

WITKIN, H. A., \& AsCH, S. E. (1948). Studies in space orientation: IV. Further experiments on perception of the upright with displaced visual fields. Journal of Experimental Psychology, 38, 762-782.

Witkin, H. A., \& Goodenough, D. R. (1981). Cognitive styles: Essence and origins. Psychological Issues, Monograph 51.

Zoccolotti, P., Antonucci, G., Goodenough, D., Pizzamiglio, L., \& SPINELLI, D. (1992). The role of frame size on upright and tilted observers in the rod and frame test. Acta Psychologica, 72, 171-187.

Zoccolotti, P., Antonucci, G., \& SPInelli, D. (1993). The gap between rod and frame influences the rod-and-frame effect with small and large inducing displays. Perception \& Psychophysics, 54, 14-19.

\section{NOTES}

1. Other distinguishing factors between direct and indirect effects refer to the spatial frequency effect (Wenderoth \& Johnstone, 1988a), the presence of different time courses (Wenderoth \& Johnstone, 1988a, Wenderoth, van der Zwan, \& Johnstone, 1989), and the differential effect of the presence of an additional external reference frame (Wenderoth \& Johnstone, 1987; Wenderoth \& Johnstone, 1988a). All of these factors are congruent with the model, which implies partially different neural mechanisms for the two effects.

2. The gap size was measured as the distance between the end of the rod and the inner edge of the top (or bottom) of the frame. In the standard RF, this distance varies with frame and rod inclination.

(Manuscript received January 18, 1994; revision accepted for publication January $3,1995$. 